\title{
DYNAMIQUE DES RELATIONS COOPÉRATIVES SUITE À UNE ACQUISITION D'ENTREPRISE
}

Nicola Mirc, Guillaume Favre

Lavoisier | « Revue française de gestion »

2016/6 N²59 | pages 121 à 138

ISSN 0338-4551

ISBN 9782746247864

Article disponible en ligne à l'adresse :

http://www.cairn.info/revue-francaise-de-gestion-2016-6-page-121.htm

\section{Pour citer cet article :}

Nicola Mirc, Guillaume Favre, « Dynamique des relations coopératives suite à une acquisition d'entreprise », Revue française de gestion 2016/6 (N²59), p. 121-138. DOI 10.3166/rfg.2016.00068

Distribution électronique Cairn.info pour Lavoisier.

(C) Lavoisier. Tous droits réservés pour tous pays.

La reproduction ou représentation de cet article, notamment par photocopie, n'est autorisée que dans les limites des conditions générales d'utilisation du site ou, le cas échéant, des conditions générales de la licence souscrite par votre établissement. Toute autre reproduction ou représentation, en tout ou partie, sous quelque forme et de quelque manière que ce soit, est interdite sauf accord préalable et écrit de l'éditeur, en dehors des cas prévus par la législation en vigueur en France. Il est précisé que son stockage dans une base de données est également interdit. 


\section{NICOLA MIRC}

IAE Toulouse, Université de Toulouse 1 Capitole, CRM - UMR 5303 CNRS

\section{GUILLAUME FAVRE}

Université de Toulouse Jean-Jaurès, LISST - UMR

5193 CNRS

\section{Dynamique des relations coopératives suite à une acquisition d'entreprise}

La recherche étudie la dynamique des relations coopératives dans la phase d'intégration organisationnelle suite à une acquisition d'entreprise. L'analyse longitudinale de l'émergence et de l'évolution de trois types de relations (collaborations, échange de connaissances et liens extraprofessionnels) entre les entreprises rapprochées montre une tendance générale à la croissance et au renforcement des relations coopératives au cours des premières années, mais révèle également une émergence séquentielle selon le type de relation ainsi qu'une logique cyclique au niveau dyadique. 
$\mathrm{L}^{\prime}$

9 intégration organisationnelle postacquisition, décrivant le processus de rapprochement de deux entreprises suite à une fusion ou acquisition, a fait l'objet d'un très grand nombre de recherches. Ces recherches ont permis d'améliorer considérablement la compréhension des facteurs organisationnels et individuels qui favorisent ou limitent les relations coopératives dans la phase post-acquisition. Toutefois ces recherches s'inscrivent largement dans une perspective soit statique, étudiant l'influence des facteurs identifiés à un instant donné, soit plutôt atomistique, analysant l'évolution des facteurs et de leur impact sur les individus et/ou entreprises de manière isolée. Les relations entre ces entreprises et les individus qui les composent, leur évolution au cours de l'intégration ne sont pas directement étudiées. Au final, on n'en sait que très peu sur la dynamique qui sous-tend la formation de liens interorganisationnels dans le cadre d'un rapprochement, permettant la combinaison et le transfert de ressources entre les deux entreprises, leur intégration (Angwin et Vaara, 2005).

Notre recherche vise à mieux comprendre cette dynamique d'un point de vue des relations coopératives entre les entreprises rapprochées. Utilisant la méthode d'analyse de réseaux, nous étudions de manière longitudinale sur une période de trois ans et demi un cas de rapprochement de deux entreprises dans le secteur du conseil. Nous appuyant sur la littérature autour des relations coopératives dans les fusionsacquisitions et la dynamique de réseaux interorganisationnels dans les partenariats interentreprises, nous étudions l'émergence et l'évolution de trois types de relations : les collaborations au travail, l'échange de connaissances et les relations extra-professionnelles. Nous investiguons la dynamique relationnelle sous trois aspects : 1) l'émergence et l'évolution des relations interentreprises dans le temps (observe-t-on des phases de croissance/de décroissance ?) ; 2) l'interdépendance des différents types de relation (un type de relation favorise-t-il l'émergence d'un autre ?) ; et 3) la stabilité des relations au niveau dyadique, donc interindividuel (les relations restent-elles stables dans le temps ou peut-on observer une tendance à la rotation des partenaires ?).

\section{I - RELATIONS COOPÉRATIVES ET INTÉGRATION ORGANISATIONNELLE POST- ACQUISITION}

Le développement de relations de coopération interentreprise a été identifié comme l'un des facteurs clés de réussite de fusionsacquisitions (Haspeslagh et Jemison, 1991 ; Angwin et Vaara, 2005). L'intégration organisationnelle doit permettre le développement de relations d'échanges ou de combinaison de ressources afin d'exploiter des synergies et complémentarités entre les deux entreprises (Larsson et Finkelstein, 1999 ; Shaver, 2006). La littérature rend compte de différents facteurs affectant le bon développement de telles relations coopératives, tels que des différences de cultures organisationnelles (Stahl et Voigt, 2008 ; Teerikangas et Irrmann, 2016), le stress et l'insécurité ressentis par les employés quant à leur avenir dans la nouvelle organisation (Buono et Bowditch, 2003 ; Monin et al., 2013 ; Rouzies, 2011) ou, sur le plan managérial, un manque d'autonomie accordée aux employés, et notamment à ceux de l'entité acquise 
(Koenig et Meier, 2001 ; Meyer et Lieb-Dóczy, 2003).

En ce qui concerne son évolution dans le temps, la vision largement répandue dans la littérature est que l'intégration organisationnelle se déroule avant tout sur la première période post-acquisition. C'est même la première année qui est considérée comme cruciale pour cadrer l'intégration et faciliter les relations interentreprises (e.g. Larsson et Finkelstein, 1999). Les différentes phases du processus d'intégration ont été, quasi exclusivement, traitées dans deux ouvrages solennels bien que peu récents : celui de Haspeslagh et Jemison (1991) et celui de Buono et Bowditch (2003). Sans proposer un cadre temporel précis, les auteurs distinguent plusieurs phases du processus. La première succédant directement la clôture de l'opération et à son annonce officielle a été caractérisée par Buono et Bowditch (2003) comme processus initial de combinaison. Elle consiste en l'élaboration « théorique » de la nouvelle organisation cible et conduit généralement à de premiers changements structurels. Le plus souvent, elle se traduit par une rationalisation des organisations. La deuxième phase consiste en l'intégration ou à la combinaison formelle des entreprises, pour reprendre le terme de Buono et Bowditch (2003). La constitution d'équipes de travail amenées à collaborer et interagir au quotidien entraînerait l'émergence de nouvelles relations de travail entre les deux entités. Parallèlement, des échanges peuvent également se développer en dehors des situations de travail, par l'estompement progressif des différences organisationnelles, du fait d'une mise en place d'un système de gestion uniforme et du développement d'une histoire commune. À terme, ce processus doit déboucher sur une amalgamation des deux organisations et la création d'une seule nouvelle entité (Haspeslagh et Jemison, 1991).

Si ces recherches ont permis une meilleure appréhension des facteurs d'influence et du déroulement d'une intégration organisationnelle dans ses grandes lignes, en fin de compte on ne sait que très peu sur la dynamique des relations coopératives la sous-tendant, de la manière dont elles émergent et se développent (Angwin et Vaara, 2005). Deux exceptions sont faites ici par les études menées par Briscoe et Tsai (2011) et Allatta et Singh (2011). Les premiers analysent le développement de collaborations dans un cabinet d'avocats $\mathrm{au}$ cours du processus post-acquisition. Ils observent que les caractéristiques des réseaux personnels pré-acquisition influencent l'apparition de liens interentreprises, mais que le développement de ces liens s'accompagne d'une baisse des liens à l'intérieur des unités rapprochées, limitant ainsi l'intégration organisationnelle. Allatta et Singh (2011) étudient l'évolution des modes de communication entre les employés au cours des trois années postacquisition. Ils observent une croissance forte des communications interentreprises immédiatement l'acquisition, puis leur nette diminution jusqu'à un stade similaire à celui précédant le rapprochement.

D'autres recherches, s'inscrivant dans le domaine des partenariats d'entreprises méritent d'être considérées ici pour nourrir nos fondements théoriques. La plupart des travaux sur la formation de réseaux interorganisationnels avancent qu'un réseau se développe de manière graduelle de relations d'échanges plutôt distancées dans un premier temps vers des relations de partenariat 
plus profonds (Ahuja, 2000 ; Ring et Van de Ven, 1994). Certains auteurs décrivent ce développement comme linéaire, avec un renforcement continu des relations, en termes de fréquence et de multiplexité (Hite, 2005; Schutjens et Stam, 2003). Dans les termes de l'analyse de réseaux, la multiplexité des relations signifie que deux individus entretiennent simultanément des relations de différentes natures (par exemple, ils travaillent ensemble mais échangent également des connaissances ou sont amis). Ces relations multiplexes sont vues comme des liens forts promouvant le transfert de ressources. Ils permettent d'accéder à des informations confidentielles (Bouty, 2000) ou complexes (Tsai, 2002 ; Hansen, 1999) et favorisent un accès plus rapide et moins coûteux aux ressources externes (Uzzi, 1997). Étudiant le processus d'intégration suite à plusieurs acquisitions, Bresman et al. (1999) ont observé un impact positif sur le transfert de savoir-faire complexes lorsque les individus étaient liés simultanément par des tâches interdépendantes et des relations de communication soutenues. Dans le cadre d'une intégration organisationnelle post-acquisition, l'émergence de relations fortes, multiplexes entre deux individus peut ainsi être associée à un niveau d'intégration plus élevé.

D'autres auteurs ont mis en avant le caractère cyclique de la formation de réseaux (Gulati et al., 2012; Marion et al., 2015 ; Ring et Van de Ven, 1994). En particulier Ring et Van de Ven (1994) proposent un modèle séquentiel avec des phases de développement et de dissolution de liens qui sont soumises à des processus formels et informels d'interprétation, d'implication et de négociation de la part des acteurs. Enfin, Gulati et al. (2012), étudiant la dynamique de partenariats dans l'industrie digitale, observent un développement en forme de U inversé, avec une émergence de partenariats croissante dans un premier temps et déclinant dans un second lorsque les entreprises commencent à être fortement interconnectées. Parmi les facteurs favorisant l'émergence de liens interorganisationnels, on peut citer la connaissance au préalable entre les partenaires, le niveau de confiance entre eux et l'existence de liens d'interdépendance.

Si ces travaux permettent de dégager de premières pistes sur la dynamique relationnelle suite à une fusion-acquisition, le sujet reste encore largement sous-exploré. En utilisant une méthodologie d'analyse de réseaux sociaux (White, 1992 ; Scott, 2000 ; Degenne et Forsé, 2004) nous cherchons à appréhender de manière tangible l'émergence et l'évolution des relations coopératives suite au rapprochement et de contribuer ainsi à une meilleure compréhension des dynamiques relationnelles sous-tendant le processus d'intégration.

\section{II - LE CAS ÉTUDIÉ}

Le cas étudié est celui d'un cabinet de conseil en recrutement qui a racheté un cabinet opérant dans le même secteur. Les deux entreprises sont des PME, l'acquéreur comprenant au moment du rachat 18 consultants, l'acquis 12. Alors que l'acquéreur et l'acquis sont spécialisés dans le secteur du conseil en recrutement, leurs offres commerciales et processus de travail varient assez fortement. Le cabinet acquéreur propose exclusivement des prestations de recrutement alors que le cabinet acquis propose des services supplémentaires d'évaluation du personnel et de formation. 
Chez l'acquéreur, les candidats pour des postes à pourvoir sont identifiés par l'approche directe tandis que le cabinet acquis emploie la méthode d'annonces.

L'acquisition a été motivée par deux objectifs principaux. D'un coté, l'expansion géographique de l'acquéreur grâce à l'implantation de l'acquis dans des zones géographiques non couvertes par le premier. De l'autre, l'exploitation du portefeuille clients cumulé des deux cabinets via, le partage de missions entre les consultants (cross-selling), et la commercialisation d'une offre combinée des prestations complémentaires des cabinets. De plus, les associés de l'acquéreur prévoyaient un échange de connaissances et de savoir-faire entre les deux entités qui permettrait à chacune d'améliorer sa performance opérationnelle et de développer les compétences de son personnel.

Après l'acquisition, les deux entreprises restent d'abord des entités séparées. Le cabinet acquis maintient son autonomie et continue à fonctionner de la même manière et avec les mêmes processus de travail qu'avant le rachat. En revanche, en vue des synergies à réaliser, le management du cabinet acquéreur encourage fortement la mise en place d'échanges et de collaborations entre les deux entreprises. Afin de favoriser leur émergence, plusieurs réunions regroupant l'ensemble du personnel sont organisées, et le cabinet racheté est relocalisé à proximité de l'acquéreur. Le mode d'acquisition réalisé correspond, par conséquent, à celui d'une acquisition de symbiose (Haspeslagh et Jemison, 1991 ; Koenig et Meier, 2001), caractérisée par une forte autonomie de l'acquis tout en développant des liens d'interdépendance entre les entreprises rapprochées au niveau opérationnel. Deux ans après le rachat, l'acquéreur procède au regroupement de l'ensemble $\mathrm{du}$ personnel dans de nouveaux locaux communs ainsi qu'à la restructuration des activités au sein d'une société mère conjointe, organisées désormais en deux grands pôles : recrutement et évaluation. D'un mode de rapprochement symbiotique, on passe alors à une absorption totale de l'entreprise acquise (Haspeslagh et Jemison, 1991).

Le cas réunit plusieurs caractéristiques qui le rendent particulièrement intéressant pour la recherche menée. Premièrement, en raison de la nature des relations coopératives induites par le projet d'acquisition, à savoir des collaborations entre consultants permettant le partage de missions et le développement d'une offre combinée, des échanges de connaissances pour favoriser le transfert de compétences et de savoir-faire, nous pouvions établir un lien direct entre les relations interindividuelles et les relations interentreprises. De plus, le savoir-faire et les processus de travail n'étant pas explicités, les relations d'échange de connaissances interindividuelles constituaient le principal vecteur pour favoriser leur transfert. Nous avons également étudié l'évolution des relations extraprofessionnelles entre les consultants des deux cabinets en raison de l'influence positive sur le partage de ressources et de connaissances qui leur est attribuée par la littérature (Tsai, 2002 ; Stahl et Sitkin, 2005). Il est à noter que les relations extraprofessionnelles sont encouragées chez l'acquéreur depuis longue date par des rencontres hebdomadaires et informelles entre consultants dans un bar pour un moment convivial après le travail. Dès le rachat, les consultants du cabinet acquis ont 


\section{METHODOLOGIE}

Afin d'étudier la dynamique des relations coopératives entre les deux entreprises après l'acquisition, nous employons la méthode d'analyse de réseaux sociaux (White, 1992 ; Scott, 2000 ; Degenne et Forsé, 2004). Nous avons collectés des données relationnelles via un questionnaire de réseaux à 6 différents moments sur une période de 3,5 ans suivant le rachat : tous les 6 mois jusqu'à la fin de la $2^{\mathrm{e}}$ année puis 1,5 an plus tard (voir figure 1) ${ }^{1}$. Le protocole de recherche développé est très similaire à celui de Burt (1992) et de Lazega (2001). Chaque questionnaire comportait une liste de tous les consultants des deux cabinets. Chaque consultant était demandé d'indiquer toutes les personnes avec lesquelles il avait été en relation durant les 6 mois précédents l'administration du questionnaire. Les relations à renseigner dans le questionnaire ont été de trois types : 1) les collaborations sur des missions de conseil ou des prospections de clients, 2) les échanges de connaissances sous forme de conseils demandés à des collègues sur des sujets liés à l'activité opérationnelle, et 3) les relations extraprofessionnelles, telles que « des verres pris ensembles après le travail » ou des relations plus amicales. Le taux de réponse varie selon les périodes entre $80 \%$ et $100 \%$.

Comme nous nous intéressons dans cet article exclusivement aux relations interentreprises, seules les relations liant deux consultants de différents cabinets ont été prises en compte. La première période d'observation portant sur la période pré-acquisition est alors exclue de l'analyse. Notons qu'aucune relation n'existait entre les consultants avant le rachat. À l'inverse des relations d'échange de connaissances et extra-professionnelles, les collaborations ont été symétrisées. Il s'agit en effet d'une relation par définition réciproque et nous avons considéré l'existence d'une collaboration seulement à partir du moment où les deux acteurs se sont mutuellement cités comme partenaires.

Les données ont été analysées avec le logiciel UCINET pour les mesures de réseaux et le logiciel SPSS pour des analyses statistiques standard. Nous utilisons ici des tests statistiques de type Khi2 pour évaluer le niveau de relations interentreprises par rapport au niveau de relations à l'intérieur de chaque entreprise ainsi que des procédures de tests QAP (Quadratic Assignment Procedure) pour les analyses de l'interdépendance des relations de différente nature afin de tenir compte de la dépendance des observations inhérente à ce type de données (Dekker et al., 2007; Krackhardt, 1988).

1. Alors que le questionnaire a été administré tous les 6 mois depuis le rachat jusqu'à P5, la dernière collecte de données (P6) a eu lieu 1,5 an plus tard. La raison à cela a été la restructuration organisationnelle opérée à la fin de P5 et la crainte d'une certaine lassitude des répondants à participer à l'étude. Pour favoriser un taux de réponse satisfaisant et pouvoir observer l'impact des changements organisationnels dans l'analyse, une collecte de données à un moment plus tardif semblait plus intéressante. Notons que le laps de temps de référence pour indiquer les relations engagées était comme pour toutes les autres périodes de 6 mois précédant la soumission du questionnaire. Le volume des relations observées est alors comparable entre les différentes périodes d'observation. 
Figure 1 - Chronologie de l'implémentation de l'acquisition et processus de collecte de données

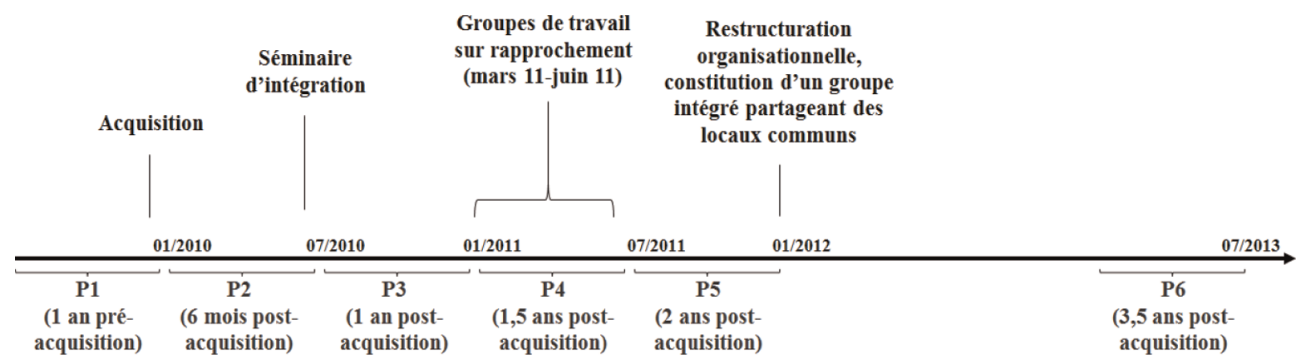

été invités à cet événement. De ce fait, il nous paraissait important d'inclure ces relations extraprofessionnelles dans les données collectées. L'observation simultanée de l'émergence et de l'évolution de relations de différente nature permet de produire une image plus complète des dynamiques relationnelles (Lazega, 2014). Un deuxième avantage de cette étude de cas relève de la taille limitée des deux cabinets qui a favorisé la collecte de données et a permis d'obtenir des taux de réponses élevés. Enfin, un troisième aspect important concerne la possibilité d'avoir pu conduire une étude longitudinale sur des réseaux entiers dès le rachat, une opportunité rare aussi bien dans la recherche sur la dynamique de réseaux que sur les fusionsacquisitions.

\section{III - RÉSULTATS}

La présentation des résultats est organisée en trois parties : 1) l'analyse de la croissance des relations de collaboration, d'échange de connaissances et extraprofessionnelles entre les entreprises rapprochées sur les trois ans et demi suivant le rapprochement ; 2) l'interdépendance des émergences de ces trois types de relations et 3) la stabilité des relations dans le temps.

\section{1. Émergence et évolution des relations coopératives dans le temps}

Le tableau 1 montre les statistiques descriptives du réseau interorganisationnel pour les trois types de relations et les cinq périodes d'observations post-acquisition.

Le nombre brut indique une croissance graduelle des trois types de relation, jusqu'à un an et demi après l'acquisition. Afin de prendre en compte la variation du nombre d'individus au cours des périodes d'observation, nous avons calculé le taux de densité interentreprises pour chaque période ${ }^{2}$. La densité est un indicateur typique pour traduire le niveau de cohésion d'un réseau. Plus la densité est élevée, plus le réseau est cohésif, ou

2. La densité interentreprise reflète le nombre de relations observées pour une période donnée par rapport au nombre de relations théoriquement possibles : $\mathrm{d}_{(\mathrm{AB})}=\mathrm{l}_{(\mathrm{AB})} / \mathrm{L}_{(\mathrm{AB})}=\mathrm{l}_{(\mathrm{AB})} /\left(\mathrm{n}_{\mathrm{A}} * \mathrm{n}_{\mathrm{B}}\right) 2$; la densité interorganisationnelle $\left.\mathrm{d}_{(\mathrm{AB})}\right)$ correspond au rapport : nombre de relations réellement observées entre l'acquéreur $\mathrm{A}$ et l'acquis $\mathrm{B} \mathrm{l}_{(\mathrm{AB})} /$ nombre de relations théoriquement possibles $\mathrm{L}_{(\mathrm{AB})}$, étant égal au nombre d'acteurs de l'acquéreur $\mathrm{A}$ x nombre d'acteurs de l'acquis B. 


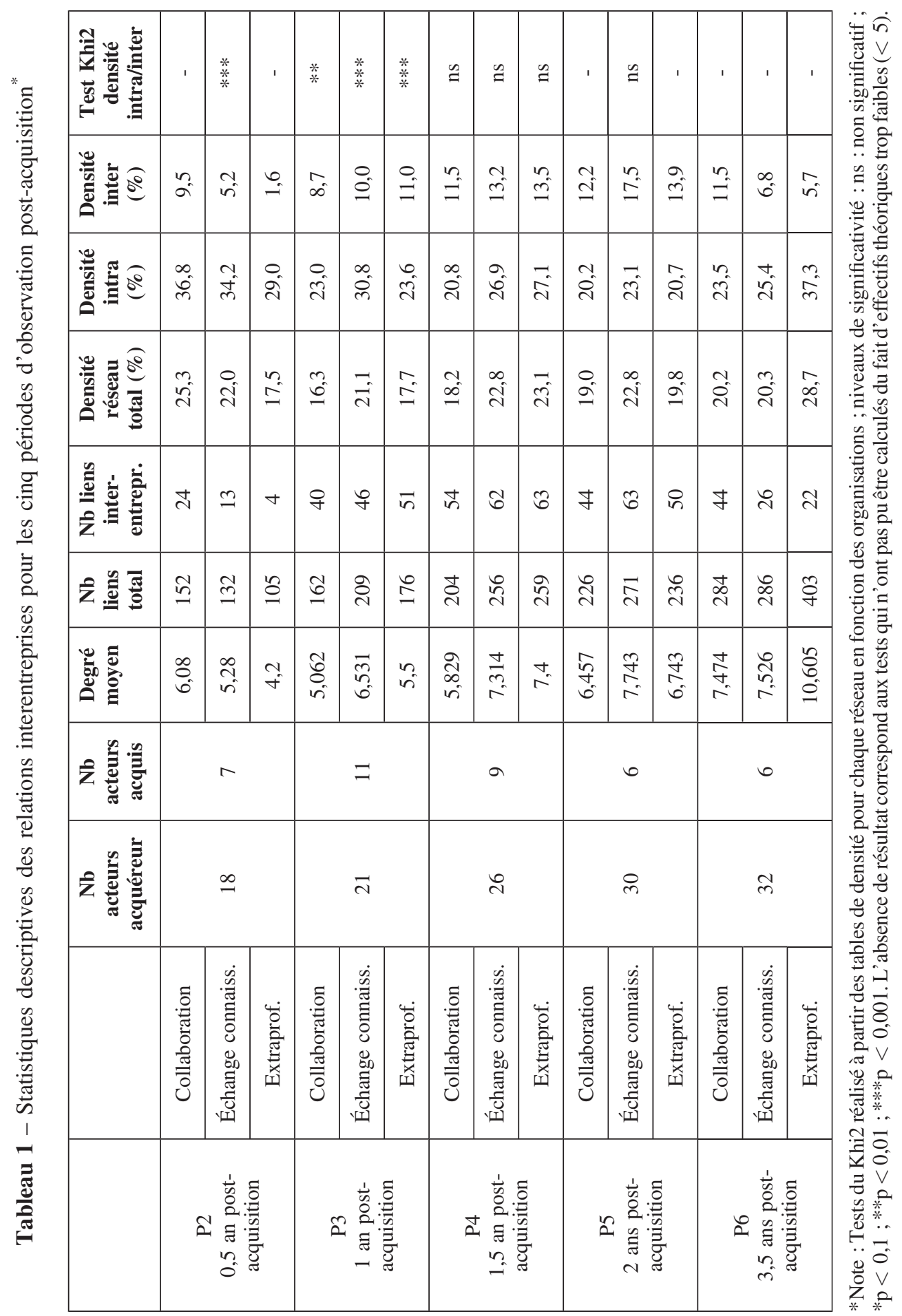


Figure 2 - Évolution des densités interentreprises par type de relation

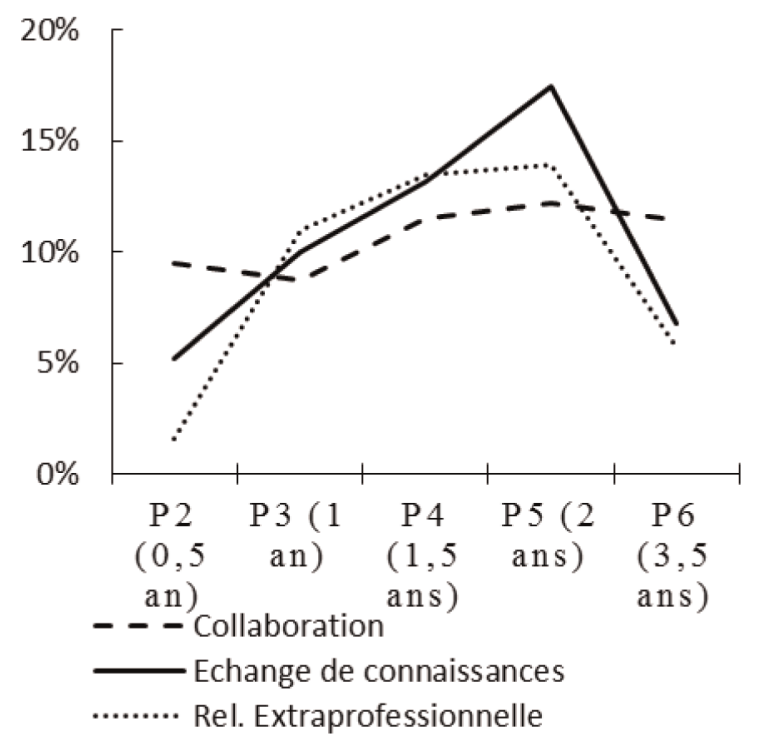

intégré. La figure 2 montre l'évolution des trois types de relations dans le temps. La densité interentreprise monte jusqu'en période 5 , donc jusqu'à deux ans après le rapprochement, et ce quel que soit le type de relation, reflétant ainsi une croissance progressive de l'intégration organisationnelle en termes de relations interentreprises. La comparaison des densités des réseaux interorganisationnels avec celles des réseaux intra-organisationnels, donc au niveau de chaque cabinet, appuie cet effet : les tests Khi2 sur les densités inter- et intra-organisationnelles $^{3}$, (voir tableau 1) montrent une tendance significative à engager des relations plutôt avec les anciens collègues qu'avec ceux du cabinet rapproché pendant la première période. Cette tendance s'estompe en période 4. Maintenant les employés collaborent, échangent des connaissances et nouent des relations extra-professionnelles indépendamment de l'entreprise d'origine. Ceci reflète une dynamique d'intégration bien avancée : un employé a ici dès la deuxième année après le rachat autant de chance de collaborer ou d'échanger des connaissances avec des membres de son entreprise d'origine qu'avec des membres de l'entité rattachée. Lorsqu'on regarde les différents types de relation, on observe que ce sont avant tout les collaborations qui émergent en premier. Leur densité est six mois après le rachat de 9,5\%, signifiant que près d'une relation sur dix théoriquement possibles est réellement activée par les consultants. Ce taux est pour la même période de $5,2 \%$ pour les échanges de connaissances et de $1,6 \%$ pour les relations extraprofessionnelles.

3. Lorsque le test est applicable puisque pour une grande partie des tests du Khi2 les effectifs théoriques sont inférieurs à 5 , ce qui ne nous permet pas de les interpréter. 
Figure 3 - Évolution des relations multiplexes

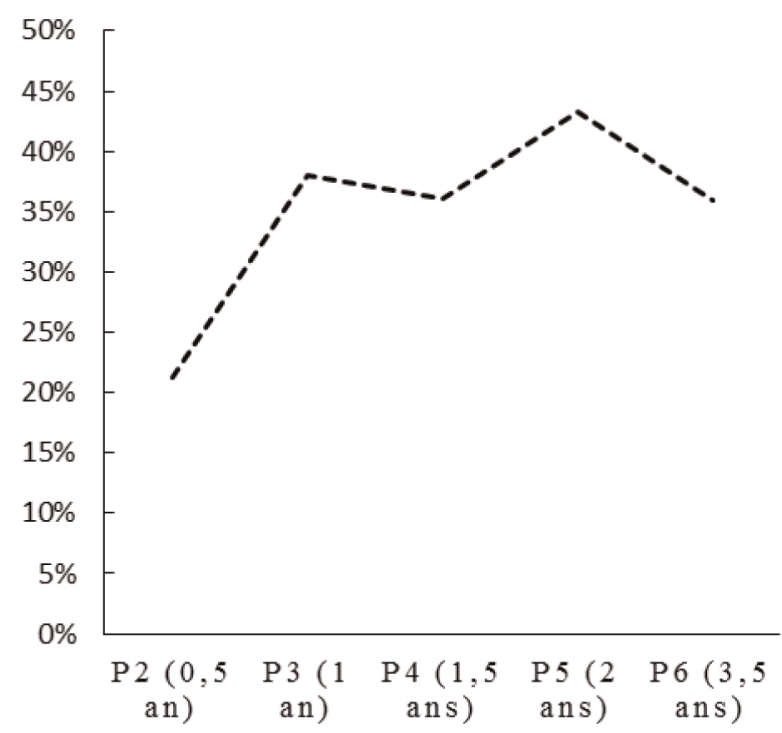

Si les collaborations au travail sont d'abord privilégiées, les relations plus informelles d'échange de connaissances et extraprofessionnelles se développent dès le second semestre post-acquisition (période 3) plus fortement. Elles connaissent, en revanche, aussi une baisse beaucoup plus significative après la deuxième année et finissent par atteindre un niveau quasi équivalent à celui de la première période après le rachat. Les collaborations augmentent dans l'ensemble progressivement et ne diminuent que légèrement en période 6 .

On observe la même tendance pour la multiplexité des relations interentreprises (figure 3), le fait que deux individus soient liés par des relations de plusieurs types. Sur les premières périodes, et surtout la première année post-acquisition, les relations multiplexes tendent à augmenter mais diminuent à leur tour également pendant la dernière période.

\section{Interdépendance des émergences de relations}

L'étude de l'interdépendance des émergences des relations coopératives (tableau 2) permet d'analyser l'ordre dans lequel se succèdent ces relations : des relations de collaborations mènent-elles à la création de relations d'échange de connaissances ? Les relations extraprofessionnelles peuventelles constituer un contexte favorisant la collaboration ?

Une première observation qui ressort assez clairement est que les corrélations quand elles existent sont toujours positives et concernent tous les types de relations. Dans l'ensemble, l'existence d'une relation en t0 favorise l'existence d'une relation en $\mathrm{t} 1$. Une collaboration peut favoriser un échange de connaissances ou une relation extraprofessionnelle, et inversement. Mais cela n'est pas le cas pour toutes les périodes, on 
Tableau 2 - L'effet de l'existence de relations interentreprises en t0 sur les relations en t1

\begin{tabular}{|c|l|l|l|}
\hline & Collaboration P3 & Échange conn. P3 & Extraprof. P3 \\
\hline Collaboration P2 & $0,515^{* * *}$ & $0,420^{* * *}$ & $0,245^{* *}$ \\
\hline Échange conn. P2 & 0,256 & $0,487^{* * *}$ & $0,213^{*}$ \\
\hline Extraprof. P2 & 0,111 & $0,379 * * *$ & $0,609 * * *$ \\
\hline & Collaboration P4 & Échange conn. P4 & Extraprof. P4 \\
\hline Collaboration P3 & $-0,004$ & 0,059 & $0,103 *$ \\
\hline Échange conn. P3 & $-0,031$ & 0,055 & 0,007 \\
\hline Extraprof. P3 & $-0,018$ & 0,036 & $0,155^{* *}$ \\
\hline & Collaboration P5 & Échange conn. P5 & Extraprof. P5 \\
\hline Collaboration P4 & $0,379 * * *$ & $0,247 * * *$ & $0,181^{* * *}$ \\
\hline Échange conn. P4 & $0,192^{* * *}$ & $0,325^{* * *}$ & $0,277^{* * *}$ \\
\hline Extraprof. P4 & $0,170^{* *}$ & $0,167 * *$ & $0,238^{* * *}$ \\
\hline & Collaboration P6 & Échange conn. P6 & Extraprof. P6 \\
\hline Collaboration P5 & 0,073 & $-0,005$ & $-0,016$ \\
\hline Échange conn. P5 & 0,051 & 0,025 & 0,024 \\
\hline Extraprof. P5 & 0,064 & 0,071 & 0,068 \\
\hline
\end{tabular}

Note : corrélations linéaires de Pearson et procédure de test QAP (Quadratic Assignment Procedure) (5 000 permutations) ; niveaux de significativité : * $\mathrm{p}<0,1 ; * * \mathrm{p}<0,01 ; * * * \mathrm{p}<0,001$

observe ici en fait une dynamique cyclique. Tous les types de relations en P2 semblent favoriser l'émergence de tous les types de relations en $\mathrm{P} 3$, et il en est de même entre les $\mathrm{P} 4$ et P5. À l'inverse, on observe des corrélations assez faibles entre les relations entre les P3 et P4 et P5 et P6. Cela signifie que quand un individu a engagé une relation avec un individu de l'autre entreprise après le rapprochement, il a tendance à maintenir cette relation aussi pendant la période suivante. Puis, quelle que soit la nature de cette relation, l'individu engagera aussi des relations de tout autre type avec ce même partenaire. Par exemple, une collaboration entraînera un échange de connaissances et des relations extraprofessionnelles, et vice versa un échange de connaissances et une relation extraprofessionnelle favorisera une collaboration. Cette dynamique multiplexe est observée lors de la première année après le rachat, puis disparaît le semestre suivant, et réapparaît l'année suivante pour disparaître de nouveau lors de la dernière période. Ce que ces résultats suggèrent est que le choix de partenaires s'opère de manière cyclique, un phénomène que nous étudions plus en détail par la suite. 
Tableau 3 - Taux de création, maintien et terminaison des relations entre deux périodes

\begin{tabular}{|c|c|c|c|c|c|}
\hline & & \multicolumn{2}{|c|}{ T0 } & \multicolumn{2}{|c|}{ T1 } \\
\hline & & $\begin{array}{l}\text { Part de liens } \\
\text { créés en t0 et } \\
\text { terminés } \\
\text { en t1 } \\
(\%)\end{array}$ & $\begin{array}{l}\text { Part de liens } \\
\text { créés en to } \\
\text { et maintenus } \\
\text { en t1 } \\
(\%)\end{array}$ & $\begin{array}{l}\text { Part de liens } \\
\text { créés en t1 } \\
\text { non existants } \\
\text { en to } \\
(\%)\end{array}$ & $\begin{array}{l}\text { Part de liens } \\
\text { maintenus } \\
\text { en t1 par } \\
\text { rapport à t0 } \\
(\%)\end{array}$ \\
\hline \multirow{3}{*}{$\mathrm{P} 2 / \mathrm{P} 3$} & Collaboration & 54,5 & 45,5 & 37,5 & 62,5 \\
\hline & Échange conn. & 46,2 & 53,8 & 53,3 & 46,7 \\
\hline & Extraprof. & 20 & 80 & 42,9 & 57,1 \\
\hline \multirow{3}{*}{$\mathrm{P} 3 / \mathrm{P} 4$} & Collaboration & 94,7 & 5,3 & 95,7 & 4,3 \\
\hline & Échange conn. & 87,5 & 12,5 & 90,6 & 9,4 \\
\hline & Extraprof. & 78 & 22 & 80 & 20 \\
\hline \multirow{3}{*}{$\mathrm{P} 4 / \mathrm{P} 5$} & Collaboration & 65,4 & 34,6 & 52,6 & 47,4 \\
\hline & Échange conn. & 63,9 & 36,1 & 63,3 & 36,7 \\
\hline & Extraprof. & 75,4 & 24,6 & 68,8 & 31,3 \\
\hline \multirow{3}{*}{ P5/P6 } & Collaboration & 88,9 & 11,1 & 87,5 & 12,5 \\
\hline & Échange conn. & 94,2 & 5,8 & 88,6 & 11,4 \\
\hline & Extraprof. & 89,3 & 10,7 & 85,5 & 14,5 \\
\hline
\end{tabular}

\section{3. Évolution des relations dyadiques et turnover relationnel}

$\mathrm{Si}$ on observe croissance des relations interentreprises sur les premières périodes post-rachat, qu'en est-t-il de l'évolution de ces relations au niveau dyadique, donc interindividuel ? Sont-elles stables dans le temps ou observe-t-on au contraire un turnover relationnel ?

Le tableau 3 présente la part des relations existantes en t0 etrenouvelées en 1 par rapport aux relations existantes en t0. $80 \%$ des relations extraprofessionnelles et $45 \%$ des collaborations réalisées en $\mathrm{P} 2$ existent encore en $\mathrm{P} 3$ contre respectivement $22 \%$ et $5 \%$ entre $\mathrm{P} 3$ et $\mathrm{P} 4$. Ces chiffres remontent entre $\mathrm{P} 4$ et $\mathrm{P} 5$ pour chuter à nouveau entre $\mathrm{P} 5$ et $\mathrm{P} 6$. Cela est d'autant plus flagrant lorsque l'on regarde la part des relations nouvellement créées par rapport à la période précédente. Entre P3 et $\mathrm{P} 4$ et entre P5 et P6 les nouvelles relations représentent plus de $80 \%$ des relations existantes alors qu'elles sont comprises entre $38 \%$ et $53 \%$ entre $\mathrm{P} 2$ et $\mathrm{P} 3$ et entre $53 \%$ et $69 \%$ entre P4 et P5. On peut ainsi observer très clairement une dynamique cyclique avec des relations plutôt courtes (un an) et alternantes. Les employés semblent suivre un processus d'essai-erreur en tentant de 
nouvelles relations avec les membres de l'autre entreprise, les abandonnent par la suite et retentent des relations coopératives avec d'autres partenaires.

\section{IV - DISCUSSION}

L'étude de la dynamique des relations coopératives post-acquisition a été guidée par trois sujets d'investigation : la structure d'émergence et d'évolution des trois types de relations observées - collaborations, échange de connaissances et relations extra-professionnelles - leurs interdépendances, et la logique dyadique les soustendant. L'analyse a permis d'identifier les tendances suivantes :

1) Globalement, nous observons une croissance des relations coopératives pendant les deux premières années après le rapprochement, avec une tendance vers des liens plus forts multiplexes.

2) Les relations de collaborations, plus formelles sont privilégiées dans un premier temps. Les relations d'échange de connaissances et extraprofessionnelles, plus informelles, émergent plus tardivement et selon une intensité variable dans le temps, passant d'une forte croissance à un déclin presque symétrique deux ans après le rachat. Et,

3) l'émergence des relations coopératives suit au niveau dyadique une logique cyclique, régie par une rotation périodique des partenaires de coopération.

\section{Des relations coopératives en croissance : une intégration organisationnelle en progression}

L'augmentation continue des relations coopératives suivant le rachat traduit un processus d'intégration tel que décrit, bien qu'en d'autres termes, par la recherche sur les fusions-acquisitions (e.g. Birkinshaw et al., 2000) et les partenariats stratégiques (e.g. Hite, 2005). L'acquisition ayant été motivée par la création de relations conjointes et d'échange de connaissances, la multiplication des liens interentreprises reflète dans cette optique une implémentation graduelle $\mathrm{du}$ projet initial. Il est intéressant de remarquer que cette croissance s'accompagne à la fois d'une baisse des relations au niveau intra-organisationnel (conformément aux observations de Briscoe et Tsai, 2011) mais aussi d'un renforcement relationnel au niveau dyadique dans le temps : les liens entre deux individus ont tendance à concerner des niveaux d'interaction différents, donc à devenir multiplexes, traduisant ainsi une progression de l'intégration organisationnelle sur le plan relationnel.

Ce qui paraît plus surprenant est la décroissance à la fois du volume et de la force des relations lors de la dernière période d'observation, donc après la deuxième année postacquisition qui, de plus, correspond à une absorption totale de l'acquis. Le regroupement des activités en pôles intégrés et l'installation du personnel dans des locaux communs devait, au contraire, permettre le renforcement des liens entre les deux entreprises et créer un contexte propice à l'émergence de relations opérationnelles et interpersonnelles. Or, non seulement le nombre de relations coopératives interentreprises baisse à cette période, mais celles-ci sont aussi moins souvent multiplexes. $\mathrm{Ce}$ résultat peut en partie résulter de l'augmentation de la taille de l'entreprise acquéreuse, atteignant en période 6 trente-deux consultants (contre dix-huit en période 2) et au fait que le nombre de collaborations par individu ne peut être infini. 
Une explication alternative peut être avancée. La tendance observée pourrait ainsi indiquer que les individus engagent surtout dans les deux premières années suivant le rapprochement des relations plus soutenues avec des membres de l'autre entreprise. Motivés par la découverte du partenaire et curieux d'explorer de nouvelles opportunités, les employés sont effectivement souvent enclins à engager des relations professionnelles ou sociales avec leurs nouveaux collègues (Teerikangas, 2012). Puis, une fois la valeur de telles relations sondée, et la première euphorie passée, les relations deviennent alors moins nombreuses, malgré le contexte organisationnel a priori favorable. On pourrait ainsi interpréter cette tendance observée comme une forme de normalisation des relations coopératives trois ans post-acquisition. Dans cette perspective, nous développons les résultats avancés par Allatta et Singh (2011), qui suggèrent une dynamique post-acquisition de croissance puis de décroissance jusqu'à une certaine normalisation du niveau de communications entre employés, en observant une dynamique similaire également pour les collaborations, échanges de connaissances et liens extraprofessionnels. Toutefois, les collaborations paraissent bien plus stables que les autres types de relations qui, eux, montrent une dynamique de croissance et de décroissance beaucoup plus soutenue.

\section{Une émergence séquentielle : des collaborations opérationnelles aux relations plus informelles}

L'analyse a montré que l'émergence des relations interentreprises est plutôt séquentielle et varie selon la nature de celles-ci. La tendance est dans un premier temps de privilégier des collaborations portant sur l'activité opérationnelle - partage de missions et commercialisation de services conjoints. Seulement dans un second temps, les relations informelles d'échange de connaissances et extraprofessionnelles émergent. Elles se développent par contre de manière plus importante sur les deux premières années post-acquisition mais diminuent ensuite aussi de façon plus forte.

Ce résultat nuance les travaux sur la progression de l'intégration organisationnelle dans le temps (e.g. Buono et Bowditch, 2003). L'émergence plus tardive de relations plus informelles peut s'expliquer par le fait que pendant les premières phases de l'intégration organisationnelle, les individus se trouvent souvent dans une situation de découverte du partenaire (Larsson et Finkelstein, 1999 ; Haspeslagh et Jemison, 1991). Ils n'ont pas ou peu de connaissances des membres de l'autre entreprise, de leurs compétences et savoirs. Or, afin de pouvoir échanger des connaissances avec une autre personne ou la contacter pour obtenir des conseils, il faut être conscient de la connaissance qu'il/elle possède (Borgatti et Cross, 2003 ; Kang et al., 2007). On peut interpréter ce résultat comme reflétant une certaine tendance à favoriser une dynamique formelle de la combinaison organisationnelle, en lien avec les observations de Buono et Bowditch (2003). Ensuite s'instaure une dynamique vertueuse, permettant aux individus de développer, grâce aux interdépendances opérationnelles créées (Sosa et al., 2015), des relations de confiance ainsi qu'une connaissance suffisante du champ de savoir du partenaire afin de le solliciter à l'avenir pour des conseils ou d'autres collaborations. 
Par contre, cette dynamique vertueuse ne suit pas une trajectoire linéaire mais est soumise à une rotation relationnelle forte de la part des individus.

\section{Une dynamique cyclique des relations dyadiques}

Si au niveau du réseau global nous avons observé une augmentation continue des relations sur les premières années, au niveau dyadique, donc interindividuel, la dynamique se présente différemment. Les relations liant deux mêmes acteurs progressent, au contraire, de façon cyclique suivant des phases de création et de disparition de liens. Ainsi, nous observons une phase de création de relations dyadiques directement après le rapprochement qui favorisent ensuite l'émergence de relations multiplexes de toute nature. Ces liens sont ensuite maintenus pendant un an et abandonnés subséquemment. De nouvelles dyades se forment alors à ce moment. De nouveau, les relations sont renforcées avec le même partenaire et maintenues pendant un an, pour être encore abandonnées par la suite.

Ce résultat contraste la vision largement répandue dans la littérature scientifique et managériale que l'intégration organisationnelle se déroule avant tout sur la première période, voire la première année postacquisition (e.g. Larsson et Finkelstein, 1999). Or, comme nous l'observons dans le cas étudié, si des relations coopératives sont bien créées durant la première période, elles ont également tendance à se transformer fondamentalement par la suite. Derrière la tendance globale à multiplier les relations avec des membres de l'entreprise partenaire au cours du temps se cache en réalité un important turn-over relationnel.
Le développement des relations dyadiques dans le cadre de cette acquisition semble ainsi s'apparenter à un processus d'essaierreur. Le développement de collaborations rapide au début de processus indique qu'il s'agit plutôt pour les individus de se lancer dans de nouveaux partenariats, sans connaissance a priori des membres de l'autre entreprise. Ensuite, après une phase d'expérimentation ou de «test» du partenaire pendant un an, la dynamique se poursuit avec un autre partenaire. Une telle dynamique cyclique a été suggérée dans le modèle séquentiel théorisé par Ring et Van den Ven (1994) sur l'émergence de relations coopératives interorganisationnelles, appliqué par exemple par Marion et al. (2015). Les auteurs avancent notamment que la formation et dissolution de liens interorganisationnels reposent sur des contrats psychologiques entre les individus les engageant. Ceux-ci négocient continuellement les termes des relations qu'ils entretiennent ce qui amène à leur évolution cyclique (émergence - expérience éventuellement maintien selon une période variable - dissolution). Dans le contexte étudié de l'intégration post-acquisition, cette dynamique reflète une certaine fragilité des relations créées dans les premières phases du rapprochement. Les premières dynamiques intégratives semblent ainsi tout sauf pérennes mais peuvent être facilement mises en cause par les individus concernés. L'intégration organisationnelle entre deux entreprises apparaît ici comme un processus qui ne se déroule pas selon un mode linéaire mais dont le niveau et la progression sont soumis au rythme des interactions entre les individus les composants. 


\section{CONCLUSION}

Dans l'ensemble, nos résultats contribuent à la littérature sur les fusions-acquisitions en proposant des éclairages sur les formes et évolutions des relations sous-tendant le processus d'intégration organisationnelle. L'analyse de données relationnelles avec une approche de réseaux sociaux nous a permis de développer une perspective à la fois plus tangible et nuancée des dynamiques intégratives en œuvre. D'un point de vue managérial, elle met l'accent sur la considération de la dynamique relationnelle comme indicateur de progression de l'intégration organisationnelle. Elle suggère également l'importance d'un management actif de ces relations, en créant un contexte favorable à leur émergence (pilotage des collaborations opérationnelles pour amorcer la coopération, stimulation de rencontres informelles pour favoriser la création de liens informels, etc.). Nos résultats sont cependant limités par le fait qu'ils ne portent que sur un cas d'étude mené. D'autres recherches similaires seront nécessaires pour confirmer les tendances observées et développer une perspective plus complète des dynamiques relationnelles en œuvre dans l'intégration organisationnelle post-acquisition.

\section{BIBLIOGRAPHIE}

Ahuja G. (2000). "Collaboration networks, structural holes, and innovation: A longitudinal study”, Administrative Science Quarterly, vol. 45, n $\mathrm{n}^{\mathrm{O}}$ 3, p. 425-455.

Allatta J.T. et Singh H. (2011). "Evolving communication patterns in response to an acquisition event", Strategic Management Journal, vol. 32, n ${ }^{\circ}$ 10, p. 1099-1118.

Angwin D. et Vaara E. (2005). "Connectivity in merging organizations: Beyond traditional cultural perspectives", Organization Studies, vol. 26, n 10, p. 1445-1453.

Birkinshaw J., Bresman H. et Håkanson L. (2000). "Managing the post-acquisition integration process: How the human integration and task integration processes interact to foster value creation”. Journal of Management Studies, vol. 37, n 3, p. 395-425.

Bresman H., Birkinshaw J. et Nobel R. (1999). "Knowledge transfer in international acquisitions", Journal of International Business Studies, p. 439-462.

Borgatti S.P. et Cross R. (2003). "A relational view of information seeking and learning in social networks", Management Science, vol. 49, no 4 p. 432-445.

Bouty I (2000). "Interpersonal and interaction influences on informal resource exchanges between R\&D researchers across organizational boundaries", Academy of Management Journal, vol. 43, n 1, p. 50-65.

Briscoe F. et Tsai W. (2011). "Overcoming relational inertia: how organizational members respond to acquisition events in a law firm”, Administrative Science Quarterly, vol. 56, $n^{\circ} 3$ p. $408-440$.

Buono A. et Bowditch J. (2003). Human side of mergers and acquisitions: Managing collisions between people and organisations, Edit. Beard Books, Washington DC.

Burt R.S. (1992). Structural holes: The social structure of competition, Harvard Univ. Press. 
Dekker D., Krackhardt D. et Snijders T.A. (2007). "Sensitivity of MRQAP tests to collinearity and autocorrelation conditions", Psychometrika, vol. 72, n 4, p. 563-581.

Degenne A. et Forsé M. (2004). Les réseaux sociaux, Éd. Armand Collin, coll. "U”, Paris.

Gulati R. et Gargiulo M. (1999). "Where do interorganizational networks come from?", American Journal of Sociology, vol. 104, $\mathrm{n}^{\circ}$ 5, p. 1439-1493.

Granovetter M. (1973). "The strength of weak ties", American Journal of Sociology, 78, p. $1360-1380$.

Gulati R., Puranam P. et Tushman M. (2012). "Meta-organization design: Rethinking design in interorganizational and community contexts", Strategic Management Journal, vol. $33, n^{\circ} 6$, p. 571-586.

Hansen M.T. (1999). "The search-transfer problem: The role of weak ties in sharing knowledge across organization subunits", Administrative Science Quarterly, vol. 44, $\mathrm{n}^{\circ} 1$, p. 82-111.

Haspeslagh P. et Jemison D.B. (1991). Managing acquisitions: creating value through corporate renewal, The Free Press, New York

Hite J. M. (2005). "Evolutionary processes and paths of relationally embedded network ties in emerging entrepreneurial firms", Entrepreneurship Theory and Practice, vol. 29, $\mathrm{n}^{\mathrm{o}} 1$, p. 113-144.

Kang S.C., Morris S.S. et Snell S.A. (2007). "Relational archetypes, organizational learning, and value creation: Extending the human resource architecture", Academy of Management Review, vol. 32, $\mathrm{n}^{\circ}$ 1, p. 236-256.

Koenig G. (1996). « Karl. E. Weick », Revue française de gestion, $\mathrm{n}^{\circ}$ 108, mars-mai, p. $57-70$.

Kœnig G. et Meier O. (2001). « Acquisitions de symbiose : les inconvénients d'une approche rationaliste»,M@n@gement, vol. 4, n 1, p. 23-45.

Krackhardt D. (1988). "Predicting with networks: Nonparametric multiple regression analysis of dyadic data", Social networks, vol. 10, n $\mathrm{n}^{\mathrm{O}}$ 4, p. 359-381.

Larsson R. et Finkelstein L. (1999). "Integrating strategic, organizational and human resource perspectives on mergers and acquisitions: a case of synergy realization", Organization Science, 10, p. 1-26.

Lazega E. (2001). The collegial phenomenon-The social mechanisms of cooperation among peers in a corporate law partnership, Oxford University Press.

Lazega E. (2014). Réseaux sociaux et structures relationnelles, PUF, Paris.

Marion T.J., Eddleston K.A., Friar J.H. et Deeds D. (2015). "The evolution of interorganizational relationships in emerging ventures: An ethnographic study within the new product development process", Journal of Business Venturing, vol. 30, $\mathrm{n}^{\circ} 1$, p. 167-184. 
Meyer K.E. et Lieb-Dóczy E. (2003). "Post-acquisition restructuring as evolutionary process", Journal of Management Studies, vol. 40, n ${ }^{\circ}$ 2, p. 459-482.

Ring P.S. et Van de Ven A.H. (1994). "Developmental processes of cooperative interorganizational relationships", Academy of Management Review, vol. 19, n 1, p. 90-118.

Rouzies A. (2011). “Antecedents of employees' identification with a merger: A threestage empirical study”, International Studies of Management \& Organization, vol. 41, $\mathrm{n}^{\mathrm{o}} 3$, p. 25-41.

Schutjens V. et Stam E. (2003). "The evolution and nature of young firm networks: A longitudinal perspective", Small Business Economics, vol. 21, no 2, p. 115-134.

Scott J. (2000). Social Network Analysis, Sage Publications, Londres.

Shaver J.M. (2006). "A paradox of synergy: contagion and capacity effects in mergers and acquisitions”, Academy of Management Review, 31, p. 962-976.

Sosa M.E., Gargiulo M. et Rowles C. (2015). "Can informal communication networks disrupt coordination in new product development projects?", Organization Science, vol. $26, \mathrm{n}^{\circ} 4$, p. 1059-1078.

Stahl G.K. et Sitkin S.B. (2005). "Trust in mergers and acquisitions", Mergers and acquisitions: Managing culture and human resources, p. 82-102.

Stahl G.K. et Voigt A. (2008). "Do cultural differences matter in mergers and acquisitions? A tentative model and examination”, Organization Science, 19, p. 160-176.

Teerikangas S. (2012). "Dynamics of acquired firm pre-acquisition employee reactions", Journal of Management, vol. 38, n 2, p. 599-639.

Teerikangas S. et Irrmann O. (2016). "Cultural change following international acquisitions: cohabiting the tension between espoused and practiced cultures", Management International Review, vol. 56, n 2, p. 195-226.

Tsai W. (2002). "Social structure of "coopetition" within a multiunit organization: Coordination, competition, and intraorganizational knowledge sharing", Organization Science, vol. 13, $\mathrm{n}^{\mathrm{o}} 2$, p. 179-190.

Uzzi B. (1997). "Social structure and competition in interfirm networks: The paradox of embeddedness", Administrative Science Quarterly, p. 35-67.

White H. (1992). Identity and Control: A Structural Theory of Social Action, Princeton University Press 